\title{
RACCORDEMENT DES FIBRES OPTIQUES à l'habitat - FTTH
}

\author{
Jean-Michel MUR \\ Président d'honneur du Club fibres \\ optiques et réseaux \\ Jm.mur@orange.fr
}

Le déploiement de la fibre optique jusqu'à l'habitat (fiber to the home - FTTH) a des conséquences (on pourrait parler de défis à relever) comme la création des fibres UIT-T G.657, une pénurie de main-d'œuvre, de nouveaux réseaux optiques passifs et des évolutions des équipements. Tour d'horizon...
T) Union internationale des télécommunications (UIT) publie des « Recommandations » pour les architectures des réseaux, câbles, procédures d'installation, protocoles, etc. La recommandation UIT-T G.652 définit les fibres optiques généralement installées par les opérateurs dans les réseaux de transport. Mais, dès le déploiement du FTTH, trois problèmes apparurent : des contraintes d'installation dont les rayons de courbure et l'encombrement dû à une forte densité de fibres dans les câbles pour un espace souvent réduit, la desserte des territoires ruraux, et une pénurie de main d'œuvre qualifiée. À partir de la fibre G.652, visitons les solutions apportées dont les fibres insensibles aux pertes par courbure UIT-T G.657.

\section{La recommandation UIT-T G.652}

L'UIT-T G.652 « Caractéristiques des câbles à fibres optiques monomodes » décrit les fibres et les attributs géométriques, mécaniques et de transmission dont le point-clé : la longueur d'onde de dispersion chromatique nulle au voisinage de $1350 \mathrm{~nm}$. Depuis leur création en 1988, elles sont installées sans discontinuer mais, pour suivre les évolutions et les besoins croissants du produit débit $\times$ distance, la neuvième version de la G.652 a été publiée en 2016. Quelques exemples schématisés par Laurent Gasca, responsable produits et normalisation des solutions télécoms de Prysmian Group : "Dans les années 1990, les améliorations ont porté sur la dispersion due à la polarisation ou PMD. Dans les années 2000, ce fut la lutte contre le pic d'absorption de l'eau avec la création de fibres à faible pic $\mathrm{OH}$ appelées low water peak (LWP) voire zero water peak (ZWP). Dansles années 2010, lesévolutions ont porté sur les diamètres de modes pour limiter les différences de dispersion d'un fabricant à l'autre et permettre ainsi une meilleure interopérabilité. »

Aujourd'hui, les fibres G.652 se répartissent en deux types : G.652.B et G.652.D, aussi connues sous les références $\mathrm{B} 1.1$ et $\mathrm{B} 1.3$ de la CEI. D'après Laurent Gasca : «Les fibres G.652.B sont la version historique, spécifiées à 1310, 1550 et 1625 nanomètres et encore installées dans quelques pays maisplus en France. Les opérateurs préferent les G.652.D qui sont caractérisées sur toute la plage des longueurs d'onde

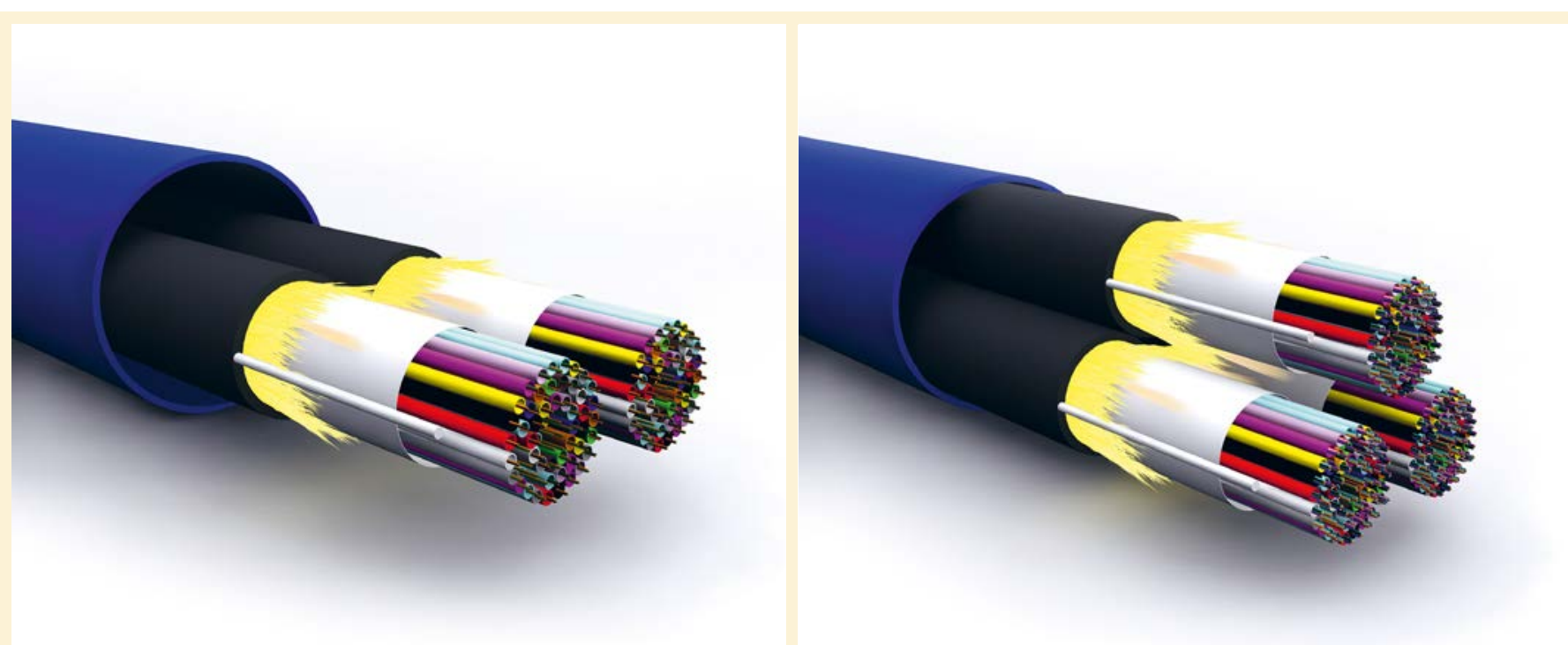

Figure 1. Exemple de densification des câbles : dans une même conduite, on peut faire passer deux câbles de 720 fibres classiques avec un revêtement de $250 \mu \mathrm{m}$ (à gauche) ou trois câbles de 720 fibres ayant un revêtement réduit à $200 \mu \mathrm{m}$, soit un gain de $50 \%$ (à droite). 


\begin{tabular}{|c|c|c|c|c|c|c|}
\hline & \multicolumn{3}{|c|}{ UIT-T G.657.A1 } & \multicolumn{3}{|c|}{ UIT-T G.657.A2 } \\
\hline Rayon (mm) & & 15 & 10 & 15 & 10 & 7,5 \\
\hline Nombre de tours & & 10 & 1 & 10 & 1 & 1 \\
\hline Perte max. à 1550 nm (dB) & & 0,25 & 0,75 & 0,03 & 0,1 & 0,5 \\
\hline \multirow[t]{2}{*}{ Perte max. à 1625 nm (dB) } & & 1 & 1,5 & 0,1 & 0,2 & 1 \\
\hline & \multicolumn{3}{|c|}{ UIT-T G.657.B2 } & \multicolumn{3}{|c|}{ UIT-T G.657.B3 } \\
\hline Rayon (mm) & 15 & 10 & 7,5 & 10 & 7,5 & 5 \\
\hline Nombre de tours & 10 & 1 & 1 & 1 & 1 & 1 \\
\hline Perte max. à 1550 nm (dB) & 0,03 & 0,1 & 0,5 & 0,03 & 0,08 & 0,15 \\
\hline Perte max. à 1625 nm (dB) & 0,1 & 0,2 & 1 & 0,1 & 0,25 & 0,45 \\
\hline
\end{tabular}

adaptive optics for

optical metrology,

lasers and microscopy

Tableau 1. Pertes maxima autorisées pour les fibres G.657 en fonction du rayon de courbure et du nombre de tours. Source : Recommandation UIT-T G.657, $4^{e}$ édition de novembre 2016, extrait des pages 16 et 17 .

de 1310 à 1625 nanomètres. » D'après les câbliers, le marché mondial annuel de tous les types de fibres serait estimé à près de 500 millions de kilomètres dont $60 \%$ en Chine suivie de l'Inde puis des États-Unis et de la France. Notre pays, à marche forcée, essaie de rattraper son retard de déploiement. Les efforts viennent des grands opérateurs et des collectivités locales via les réseaux d'initiative publique. Cette forte demande a pour corollaire une tension sur les approvisionnements. Le syndicat professionnel Sycabel estime que, pour y répondre, les industriels ont investi en France plus de 210 millions d'euros ces six dernières années. Àla question: "Que se passera-t-il pources investissements lorsque le FTTH couvrira la France? ", Laurent Gasca est optimiste : "Il y aura des marchés relais vers d'autres pays plus en retard que la France sur le déploiement du FTTH et pourl'interconnexion des milliers de futures stations de base radio de la 5G» [1].

\section{La recommandation UIT-T G.657}

L'installation dans les réseaux d'accès et les immeubles se heurte aux problèmes de rayons de courbure et de place dans les chambres de tirage et les colonnes techniques d'immeubles. Aussi, un nouveau type de fibres a été créé et défini dans la recommandation UIT-T G.657 « Caractéristiques des câbles et fibres optiques monomodes insensibles aux pertes par courbure pour les réseaux d'accès", publiée fin 2006. Cette version préconisait une performance fortement améliorée en cas de courbure, comparée à celle des câbles et fibres G.652. Pour cela, deux catégories de fibres ont été adoptées : celles de catégorie A, entièrement compatibles avec les fibres G.652, pouvant aussi être utilisées dans d'autres parties du réseau, et celles de catégorie $B$, pas nécessairement compatibles avec les G.652, mais présentant de faibles valeurs de perte par macrocourbure avec de petits rayons de courbure, essentiellement destinées à l'intérieur de bâtiments.

Depuis, la G.657 a évolué jusqu'à la quatrième version publiée en 2016 avec ses quatre variantes G.657.A1, A2, B2, B3, aussi référencées B6_a1, B6_a2, B6_b2, B6_b3 par la CEI, déterminées, entre autres, par le niveau des pertes en fonction du rayon de courbure (cf. tableau 1). Ces fibres jouent leur rôle dans la distribution du point de mutualisation en pied d'immeuble jusqu'au dispositif de terminaison dans l'habitat. Cependant, Laurent Gasca complète : "Je constate une tendance lourde dans le déploiement: dans les réseaux de transport, du noud de répartition optique au point de mutualisation, les fibres G.652.D laissent leur place aux G.657.A2 qui leur sont parfaitement compatibles et apportent l'avantage d'un coefficient de courbure amélioré. »

Une raison complémentaire est la diminution de la taille du revêtement des fibres. Le diamètre de $250 \mu \mathrm{m}$ à l'origine a été réduit à $200 \mu \mathrm{m}$ il y a une dizaine d'années sous l'impulsion de Prysmian, suivi d'OFS, Corning, etc., afin de gagner en compacité dans
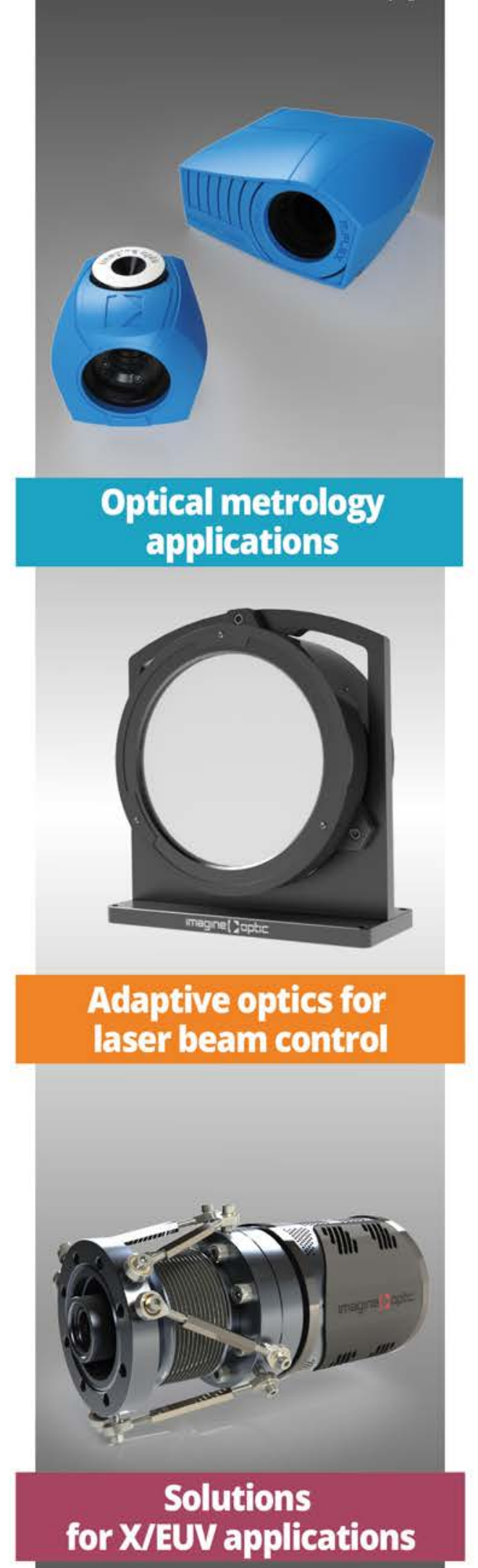

Contact us for more details: contact@imagine-optic.com or +33164861560 


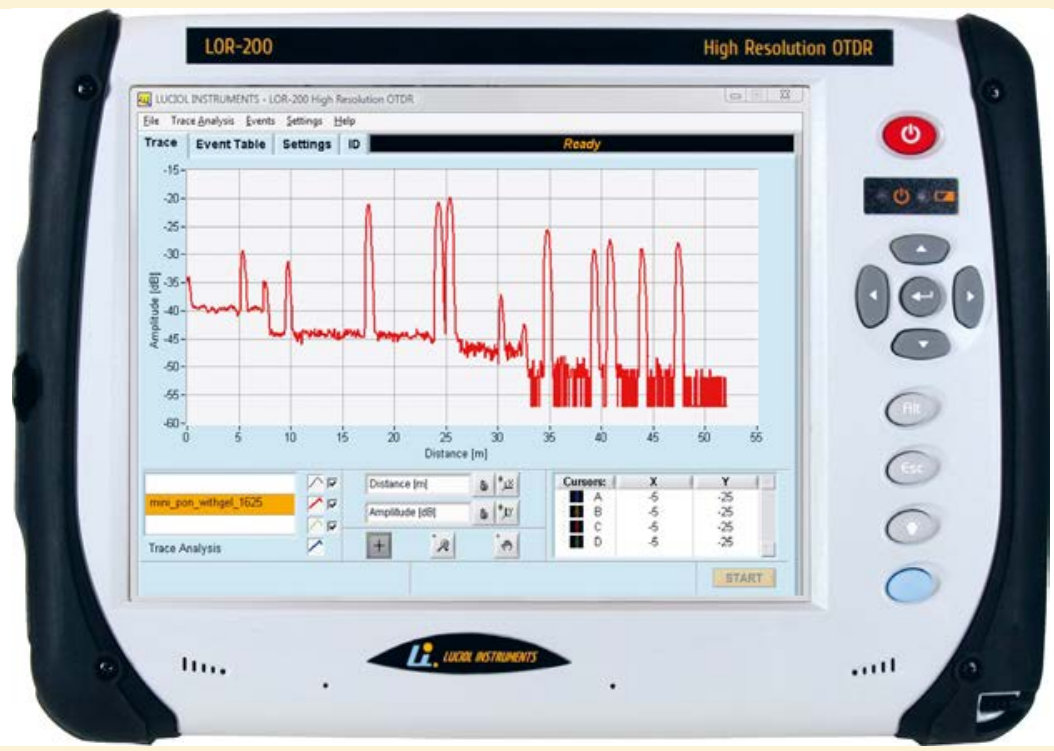

Figure 2. Un exemple d'OTDR pour contrôler les diverses branches d'un réseau optique passif. Source : Luciol Instruments.

les câbles (cf. figure 1). Cette nouvelle dimension a été intégrée aux recommandations UIT. Mais, Laurent Gasca tempère : «En pratique, la diminution de l'épaisseur du revêtement s'accompagne d'une angmentation de la sensibilité aux micro-courbures. L'utilisation de fibres insensibles aux courbures permet de contrebalancer cette dégradation. Ce revêtement de 200 um est surtout utilisé avec les fibres G.657.A1 et $A 2$ ». Ceci amène un plus faible diamètre du câble pour un nombre de fibres donné, et donc une installation plus aisée, ou bien un plus grand nombre de fibres pour un diamètre de câble donné et donc une installation moins onéreuse.

\section{Ruiralité et FTTH}

La notion d'installation moins onéreuse est prégnante lorsqu'il faut déployer le FTTH dans les zones rurales à faible densité de population. Pour Merrion Edwards, directrice du développement de la technologie et des marchés fibre optique et câbles chez Corning Optical Communications, ce déploiement présente deux aspects complémentaires : la desserte en FTTH et les nouvelles applications que sont la 5G et l'Internet des objets (IdO ou Internet of things - IoT). Pour le FTTH, Merrion Edwards préconise : «...les connexions par câbles sont intrinsèquement plus longues en milieu rural, pouvant atteindre vingt kilomètres, etles points d'accès sont moins fréquents. Ainsi, la sensibilité aux courbures entre moins en ligne de compte que l'atténuation des fibres ou l'efficacité d'installation des câbles. En utilisant une fibre à faible atténuation à la place d'une fibre standard, il est possible d'angmenter la longueur des connexions de $10 \%$ et la couverture géographique de $20 \%$, réduisant ainsi les coûts et délais de déploiement du réseau. »

Une même fibre optique peut transmettre simultanément des « services » différents, ce qui fait dire à Merrion Edwards : "...l'arrivée de la $5 \mathrm{G}$ comme vecteur de la connexité mobile haut débit contribue à la mutation du FTTH. Dorénavant, le réseau de lignes fixes à baut débit devra desservir aussi bien les antennes que les particuliers et entreprises» et elle tient à préciser: "Une fois la convergence des réseaux fixes et mobiles haut débit réalisée, une nouvelle ère dans l'bistoire de la communication sera à notre portée : l'Internet des objets. Cela differe des réseaux de communication traditionnels en ce que l'accent est mis sur la couverture géographique plus que sur celle des clients enx-mêmes. Ainsi dans les pays favorisant les déploiements FTTH en milieu rural, à l'instar de la France, il existe désormais la possibilité d'installer un réseau de lignes fixes qui connectera également les objets, comme les voitures sans chauffeur, dans l'avenir."

\section{Pénurie \\ de main d'oeuvre}

Les améliorations dans la souplesse et la facilité relative pour l'installation de la fibre ne sauraient occulter le manque de main-d'œuvre qualifiée. Marc Leblanc, directeur commercial des solutions télécoms en Europe du Sud chez Prysmian Group, s'exprime au titre de président d'Objectiffibre: «Le déploiement du plan France Très Haut Débit est un véritable défi car il faut développer des réseaux en fibres optiques pérennes et de qualité, dans un délai court. Cela nécessite de trouver puis de former les bonnes personnes au bon moment et an bon endroit» et confirme: «Le ministère du Travail et l'Agence du numérique estiment que le déploiement du FTTH va nécessiter 22000 emplois dont 12000 à pourvoir d'ici 2021, soit 12000 personnes à former pour installer 2 à 3 millions de prises par an."

Pour répondre à cela, Marc Leblanc détaille: «Objectiffibre est très investi surle sujetet travaille sur trois axes: information, formation et professionnalisation. Information, car les entreprises et le grand public sont peu au fait des métiers en question. Aussi, nous avons dressé une cartographie des fonctions à assurer et nous communiquons d'autant plus que les nouveaux embauchés peuventespérer une progression de carrière rapide car la croissance actuelle est à deux chiffres. » Pour la formation, Objectiffibre a conclu avec le ministère du Travail et les représentants de la filière industrielle un accord-cadre

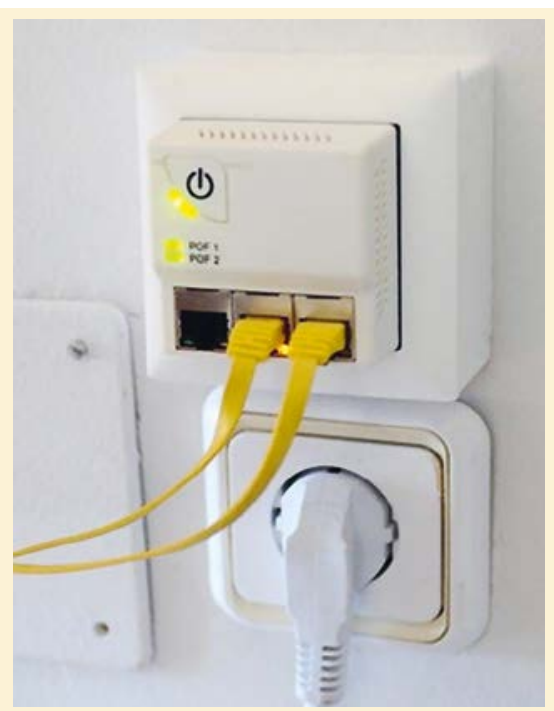

Figure 3. Dispositif de terminaison optique dans l'habitation pour fibre optique en plastique. Source : Telefónica. 
d'engagement pour le développement de l'emploi et des compétences (EDEC) et a édité un guide de référencement des organismes de formation disposant d'un plateau technique adapté et proposant des formations certifiantes ou qualifiantes. Marc Leblanc conclut : «Pourla démarche professionnalisation, nous éditons des guides d'installation des réseauxen fibres optiques. Ils ont pour but d'harmoniser les bonnes pratiques et de déployer un réseau THD de qualité. » À noter qu'ils sont mis à jour régulièrement et disponibles en téléchargement gratuit sur le site d'Objectiffibre.

Pour terminer, citons d'autres changements dus au déploiement du FTTH: les réseaux optiques passifs, les équipements, les fibres en plastique... et la «société gigabitaire».

Réseau optique passif - Connu sous l'acronyme PON (passive optical network), c'est une architecture desservant des dizaines d'abonnés à partir d'une seule fibre. Cela a débuté en 1990, avec A-PON (asynchronous transfer mode-PON), puis continué via l'accroissement du débit descendant et/ou remontant, l'allongement en distance couverte, la croissance en nombre d'abonnés desservis, le multiplexage en longueurs d'onde... En 2015 sont arrivées les recommandations UIT sur le NG-PON2 (2nd next generation PON) et le MW-PON (multiple wavelength PON). Attention, plus le nombre d'abonnés desservis par un PON augmente, plus le débit moyen par abonné diminue en proportion. Depuis 2018, des études sont en cours pour transmettre plus de $10 \mathrm{Gbit} / \mathrm{s}$ par longueur d'onde et pour le «Super-PON » qui pourrait desservir 1024 abonnés jusqu'à $50 \mathrm{~km}$ du central. À suivre...

\section{Équipements d'installation -} Exemples de changements:les soudeuses pour fibres optiques sont, généralement, destinées à souder deux fibres de type identique. La diversité G.652 et G.657 dans les réseaux a amené les fabricants à développer des soudeuses réalisant des épissures par fusion entre ces deux types de fibres. De leur côté, les réflectomètres (optical time domain reflectometer-OTDR) ont suivi deux évolutions : des modèles plus petits et portatifs à utiliser dans des cages d'escalier, et des modèles dédiés aux PON capables de différencier les mesures sur chaque branche du réseau (cf. figure 2).

Fibre optique en plastique - Fin 2017, l'opérateur espagnol Telefónica a testé l'installation de fibres optiques en plastique (FOP ou plastic optical fiber - POF) dans un réseau domestique. Les essais ont été menés avec une dorsale en FOP - câble de 2,2 $\mathrm{mm}$ de diamètre et fibre de $1 \mathrm{~mm}$ de cœur - combinée à du WiFi et des puces de la société KDPOF. Telefónica indique que la FOP est d'installation aisée ne nécessitant qu'un simple cutter. Le résultat est une installation au coût nettement réduit par rapport à une fibre en silice (cf.figure 3) .
Société gigabitaire - Le débit de $100 \mathrm{Mbit} / \mathrm{s}$ par abonné est, désormais, considéré comme tout juste suffisant. Déjà en 2005, l'opérateur Hong Kong Broadband Network (HKBN) a proposé le FTTH à $1 \mathrm{Gbit} / \mathrm{s}$. Il a été suivi par la Corée du Sud, Singapour, etc. En Europe, la volonté d'aller vers la «Gigabit society» a été affichée en 2014 par l'association ELFO (Europe's local fiber operators) et, en 2016, par le FTTH Council Europe, les États-Unis d'Amérique allant dans le même sens. À ce jour, il y aurait 150 opérateurs avec une offre à $1 \mathrm{Gbit} / \mathrm{s}$ ou plus. Le mot de la fin sera laissé à Ana Pesovic, directrice du marketing de Fixed Networks Nokia: «Itseems that the time for gigabit services to take off is upon us».

\section{POUR EN SAVOIR PLUS}

[1] « Fibre optique et $5 \mathrm{G}$ : unies pour le meilleur », MUR Jean-Michel, Photoniques n ${ }^{\circ}$ 92, pages 38 à 41, https://dol.org/10.1051/photon/20189238

\section{POUR EN SAVOIR PLUS SUR LE FITH}

\section{AFNOR}

Nombreuses normes du domaine des fibres optiques, dont NF EN 60793-2-40 de mai 2017 sur les FOP A4x.

https://www.boutique.afnor.org/xml/1910310/false

\section{ARCEP}

Autorité administrative assurant la régulation des communications électroniques et des postes, publiant des textes administratifs et législatifs sur les communications et réseaux. https://www.arcep.fr/

\section{CEI}

La norme CEI 60793-2-50:2018 sur les fibres optiques s'achète à : https://webstore.iec.ch/publication/64380

\section{FTTH Council Europe.}

Association européenne pour le développement du FITH en Europe. Plusieurs guides en téléchargement dans la section « Ressources » de : https://www.ftthcouncil.eu/

\section{Objectif fibre}

Groupement de cinq organisations et de partenaires institutionnels pour référencer des centres de formation et éditer des guides de bonnes pratiques. cf. https://www.objectif-fibre.fr/

\section{Plan France Très Haut Débit}

Volonté gouvernementale de couvrir l'intégralité du territoire en très haut débit d'ici 2022. cf. http://www. francethd.fr/

\section{SYCABEL.}

Organisation professionnelle de l'industrie des fils et câbles électriques et de communication en France. Nombreuses informations à https://www.sycabel.com/

\section{UIT-T}

Les recommandations UIT sont payantes à leur sortie mais téléchargeables gratuitement six mois après leur publication. C'est le cas des recommandations G.652 - https://www.itu.int/rec/T-REC-G.652/en - et G.657 -

https://www.itu.int/rec/T-REC-G.657/en 